\title{
Susceptibility, phenotypes of resistance, and extended-spectrum $\beta$-lactamases in Acinetobacter baumannii strains
}

\author{
Pawel Sacha ${ }^{1 *}$, Piotr Wieczorek ${ }^{1 *}$, Dominika Ojdana1, Slawomir Czaban², \\ Wioletta Kłosowska ${ }^{3}$, Anna Jurczak ${ }^{3}$, Elzbieta Tryniszewska ${ }^{1}$ \\ ${ }^{1}$ Department of Microbiological Diagnostics and Infectious Immunology, \\ Medical University of Bialystok, Poland \\ ${ }^{2}$ Department of Anesthesiology and Intensive Therapy, Faculty of Health Sciences, \\ University Hospital of Bialystok, Poland \\ ${ }^{3}$ Department of Microbiological Diagnostics and Infectious Immunology, \\ University Hospital of Bialystok, Poland \\ *Equal contribution of both authors
}

\begin{abstract}
Acinetobacter baumannii plays an increasing role in the pathogenesis of infections in humans. The bacilli are frequently isolated from patients treated in intensive care units. A growing resistance to antibiotics is leading to the emergence of strains that are multidrug-resistant and resistant to all available agents. The objective of this study was to assess susceptibility to antibiotics and to determine the presence and current level of the extended-spectrum $\beta$-lactamases (ESBLs) and attempt to isolate the Acinetobacter baumannii strain carrying the bla $a_{\mathrm{PER}}$ gene. A total of 51 strains of A. baumannii identified by phenotypic features were examined. That the strains belonged to the species was confirmed by the presence of the $b l a_{\text {OXA-51-ike }}$ gene. A broth microdilution method was used for antibacterial susceptibility testing. The occurrence of ESBLs was determined using phenotypic double-disk synergy tests. The PCR technique was used to confirm the presence of the $b l a_{\text {PER-1 }}$ gene encoding ESBL. The most active antibiotics were meropenem, cefepime and ampicillin/sulbactam, with susceptibility shown by $76.5 \%, 60.8 \%$ and $56.9 \%$ of the strains, respectively. The strains exhibited the highest resistance $(>75 \%)$ to piperacillin, tetracycline, ciprofloxacin and cefotaxime. Phenotypic tests revealed ESBL mechanism of resistance in approximately $20 \%$ of Acinetobacter baumannii isolates. However, the PCR technique did not confirm the presence of the bla $a_{\mathrm{PER}-1}$ gene in any of the Acinetobacter baumannii strains examined in our hospital. Acinetobacter baumannii strains demonstrate considerable resistance to many groups of antibiotics. Our findings indicate the involvement of enzymes belonging to families other than PER $\beta$-lactamase in resistance to $\beta$-lactams in A. baumannii. (Folia Histochemica et Cytobiologica 2012; Vol. 50, No. 1, pp. 46-51)
\end{abstract}

Key words: Acinetobacter baumannii, susceptibility to antibiotics, extended-spectrum $\beta$-lactamase

\section{Introduction}

In recent decades, Acinetobacter baumannii has become more prevalent as an opportunistic pathogen,

Correspondence address: P. Sacha, Department of

Microbiological Diagnostics and Infectious Immunology

Medical University of Bialystok,

Waszyngtona Str. 15a, 15-269 Bialystok, Poland;

tel./fax: + 48857468571 ;

e-mail: sachpt@umwb.edu.pl especially as a multi-drug resistant agent (MDR) using different mechanisms of resistance [1-3]. $\beta$-lactamases have the essential participation in the resistance to $\beta$-lactams. Acinetobacter baumannii strains producing PER-1 $\beta$-lactamase have been described in Turkey, Korea and France [4-6].

The objective of this study was to isolate Acinetobacter baumannii strains producing the PER family extended spectrum $\beta$-lactamase in patients from the north eastern of Poland, and to assess their suscepti- 
bility to antibiotics, with the determination of resistance phenotypes. Until now, the occurrence of this enzyme in Acinetobacter baumannii strains in Poland has not been reported.

The PER- 1 extended spectrum $\beta$-lactamase represents class $\mathrm{A} \beta$-lactamases classified according to the scheme of Ambler. The enzyme is weakly related to other extended spectrum $\beta$-lactamases [7]. It conditions resistance to penicillin, cefotaxime, ceftazidime and aztreonam, and its activity is well inhibited by clavulanic acid, sulbactam and tazobactam $[7,8]$. Currently, there are seven ESBL variants in the PER family [9]. PER-1 was first identified in 1993 in a $P$. aeruginosa strain isolated from a Turkish patient in France [7]. The strains have also been found in Belgium [10] and Italy [11].

\section{Material and methods}

Bacterial strains. The study material consisted of 51 Acinetobacter baumannii strains isolated from clinical samples obtained from patients treated in departments of the University Hospital in Bialystok. The bacteria were identified by the VITEK 2 GN card and the automatic system VITEK 2 (bioMerieux, Durham, NC, USA) according to the manufacturer's instructions. Additionally, advantage was taken of the ability of Acinetobacter baumannii to grow at a temperature of $44^{\circ} \mathrm{C}$ and of the presence of bla $a_{\text {OXA-51-like }}$ carbapenemase genes that are naturally found in this species [12].

Detection of $\beta$-lactamases. The detection of extended spectrum $\beta$-lactamases was performed using a standard doubledisk synergy test (DDST) [13], with disks containing ceftazidime, cefotaxime and additionally aztreonam and cefepime [14]. They were tested simultaneously on two media: (1) Mueller-Hinton agar (Oxoid Ltd, Basingstoke, UK) according to the classical double disk test [13] and (2) Mueller-Hinton agar (Oxoid) with the addition of cloxacillin (Sigma-Aldrich, St Louis, MO, USA) at a concentration of 250 $\mu \mathrm{g} / \mathrm{ml}$ [15]. Klebsiella pneumoniae ATCC 700603 strain was used as the ESBL producing control strain.

Assessment of drug-susceptibility. A broth microdilution method was used to assess susceptibility to antibiotics [16]. The minimum inhibitory concentrations (MICs) were determined within the range of $0.032-512 \mu \mathrm{g} / \mathrm{ml}$ for the following antibiotics: ampicillin/sulbactam (SAM), piperacillin (PIP), piperacillin/tazobactam (TZP), ceftazidime (CAZ), cefotaxime (CTX), cefepime (FEP), imipenem (IMP), meropenem (MEM), tetracycline (TE), gentamicin (GM), netilmicin (NET), amikacin (AN), and ciprofloxacin (CIP). Escherichia coli ATCC 25922 and Escherichia coli ATCC 35218 were used as reference strains. Following the incubation period, the MIC values of the antibiotics being studied were read for the Acinetobacter baumannii strains and susceptibility was interpreted according to the criteria established by the Clinical and Laboratory Standards Institute [17]. Drug-resistance phenotypes of the respective strains were determined.

Genetic methods. Acinetobacter baumannii genomic DNA was isolated using a Genomic Mini kit (A\&A Biotechnology, Poland). Genes were detected by the PCR technique. The PCR reaction was conducted in the final volume of $25 \mu \mathrm{l}$ assay mixture, containing $12.5 \mu \mathrm{l}$ of $2 \times$ PCR RED (DNA-Gdansk, Poland), $3 \mu$ l of extracted DNA, and $10 \mathrm{pmol}$ of each of the starters (PERf 5'-AGTCAGCGGCTTAGATA-3'; PERr 5'-CGTATGAAAAGGACAATC-3' [18], in a Cyclone 96 (PEQLAB Biotechnology GmbH, Germany) following the procedure: 1 cycle at $94^{\circ} \mathrm{C}$ for $5 \mathrm{~min} ; 35$ cycles at $94^{\circ} \mathrm{C}$ for $1 \mathrm{~min}$, at $57^{\circ} \mathrm{C}$ for $1 \mathrm{~min}$, at $72^{\circ} \mathrm{C}$ for $1 \mathrm{~min} ; 1$ cycle at $72^{\circ} \mathrm{C}$ for $10 \mathrm{~min}$ [19]. PCR products were analyzed using horizontal agarose gel electrophoresis $(1.5 \%)$. The resultant electrophoretic distributions - the expected amplification product of $978 \mathrm{bp}$ - were assessed in ultraviolet radiation (UV) and then archived using the gel documentation system ChemiDoc XRS (Bio-Rad, Hercules, CA, USA). Acinetobacter baumannii LMG 1025 strain was used in the study as a control.

\section{Results}

A total of 51 Acinetobacter baumannii strains were identified and examined. Most (78.0\%) of these strains were obtained from patients treated in the intensive care unit. Approximately $75 \%$ of these strains were isolated from samples collected from the respiratory tract, mostly from bronchial secretion (52.9\%).

The ESBL type $\beta$-lactamases were determined based on phenotypic tests. The minimum and difficult to interpret effects of inhibition zone extension around the cephalosporin disks towards the inhibitor were observed only in the test performed on Mueller-Hinton agar with cloxacillin, which inhibits chromosomal cephalosporinases that mask simultaneous occurrence of ESBL. Despite an ambiguous ESBL test pattern, approximately $20 \%$ of the strains can be treated as positive.

However, molecular biology techniques (PCR), which confirmed the presence of $\beta$-lactamase, failed to show the $b l a_{\mathrm{PER}-1} \beta$-lactamase gene in any of the Acinetobacter baumannii strains studied.

Figure 1 presents the overall susceptibility of Acinetobacter baumannii strains to the antibiotics studied. Most strains were susceptible to meropenem (76.5\%), cefepime $(60.8 \%)$ and ampicillin combined with sulbactam (56.9\%). At the same time, the lowest levels of resistance were found with meropenem and ampicillin with sulbactam $(13.7 \%)$ and piperacillin combined with tazobactam (17.6\%). About 50\% of the strains were susceptible to netilmicin, which 


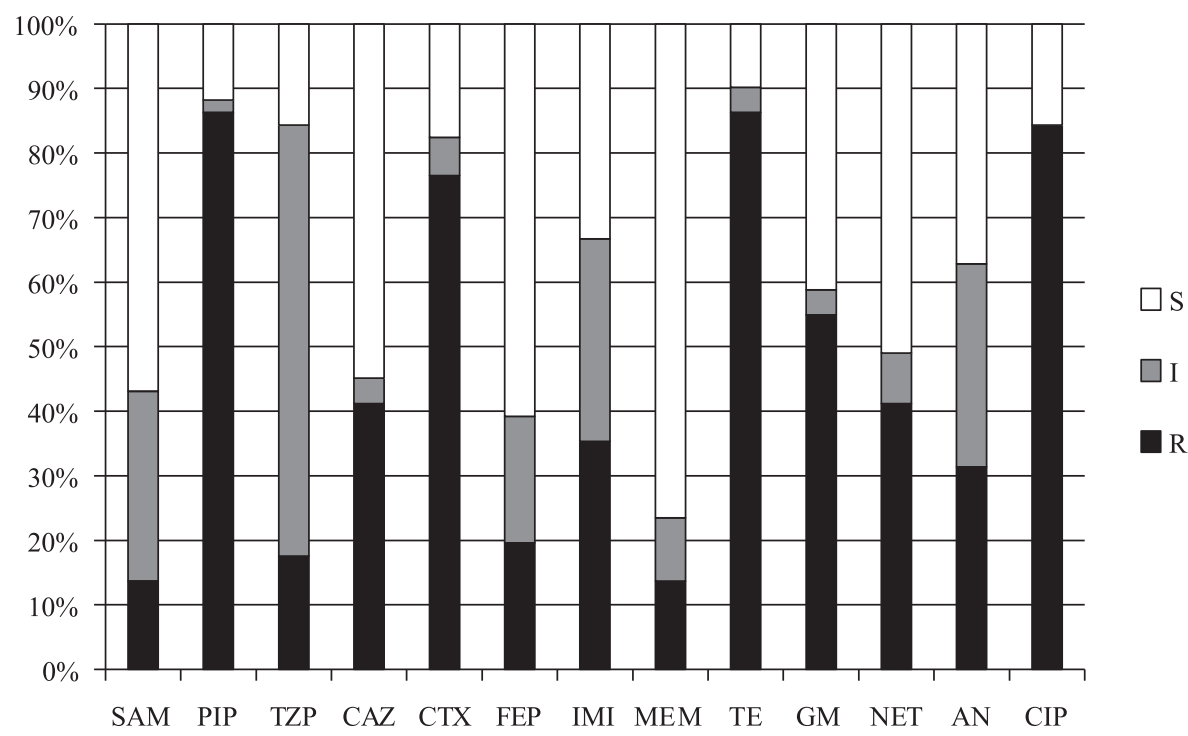

Figure 1. In vitro susceptibility of Acinetobacter baumannii strains ( $\mathrm{n}=51)$. SAM — ampicillin/sulbactam; PIP — piperacillin; TZP — piperacillin/tazobactam; CAZ — ceftazidime; CTX — cefotaxime; FEP — cefepime; IMI — imipenem; MEM — meropenem; TE — tetracycline; GM — gentamicin; NET — netilmicin; AN — amikacin; CIP — ciprofloxacin; R — resistant; I — intermediate; S — susceptible

was the most active antibiotic among the aminoglycosides examined. A considerable percentage of the strains showed intermediate susceptibility to piperacillin with tazobactam $(66.7 \%)$. The highest level of resistance was noted to piperacillin, tetracycline, ciprofloxacin and cefotaxime.

The distribution of antibiotic MICs for Acinetobacter baumannii strains is presented in Table 1. Among the most active antibiotics (meropenem, cefepime, ampicillin/sulbactam), the commonest MIC values were as follows: meropenem $\mathrm{MIC}=4$ $\mu \mathrm{g} / \mathrm{ml}$ in $41.3 \%$, cefepime $\mathrm{MIC}=4 \mu \mathrm{g} / \mathrm{ml}$ in $23.5 \%$, and ampicillin/sulbactam MIC $=16 \mu \mathrm{g} / \mathrm{ml}$ in $29.4 \%$ of the strains. In the group of the least active antibiotics, the commonest MIC values were: piperacillin MIC $\geq 256 \mu \mathrm{g} / \mathrm{ml}$ in $62.7 \%$, tetracycline MIC $=64$ $\mu \mathrm{g} / \mathrm{ml}$ in $21.6 \%$, ciprofloxacin MIC $=16 \mu \mathrm{g} / \mathrm{ml}$ in $49.0 \%$, and cefotaxime MIC $=64 \mu \mathrm{g} / \mathrm{ml}$ in $29.4 \%$ of the strains.

Based on the resistant strain category, phenotypes of resistance were determined, of which the commonest among Acinetobacter baumannii strains are shown in Table 2. Most strains were resistant to six or seven antibiotics (nine strains in each group), which in both groups presented various resistance phenotypes. The commonest profile of resistance was: $\mathrm{PIP}^{\mathrm{R}}, \mathrm{CAZ}^{\mathrm{R}}$, $\mathrm{CTX}^{\mathrm{R}}, \mathrm{TE}^{\mathrm{R}}, \mathrm{CIP}^{\mathrm{R}}$, which was observed in six strains. No strain was found to be resistant to all the antibiotics (13). Two $A$. baumannii isolates showed resistance to the largest number of antibiotics (11), whereas another two strains exhibited resistance only to tetracycline and amikacin.

\section{Discussion}

The phenotypic tests used to detect extended spectrum $\beta$-lactamases in 51 Acinetobacter baumannii strains indicate their potential occurrence in approximately $20 \%$ of isolates. When compared to literature data, this percentage seems reasonable, and the relatively high level may in most cases result from the plasmid nature of the enzymes and a selective pressure due to excessive use of antibiotics. Irrespective of the family, extended spectrum $\beta$-lactamases have been found in approximately $6.5-28 \%$ of isolates [20, 21]. Routine detection of Acinetobacter spp. strains producing ESBL using phenotypic methods may be difficult, because the effects of synergy between cephalosporins and clavulanic acid, typically observed in ESBL-positive rods of Enterobacteriaceae, can be minimal [21].

The $b l a_{\text {PER-1 }}$ gene is widespread in the strains of Acinetobacter spp., P. aeruginosa, Salmonella enterica serowar Typhimurium and Providencia rettgeri in Turkey $[9,22]$, in the region where PER-1 enzyme is present even in 32\% of Acinetobacter spp. and 55\% of $P$. aeruginosa [22]. A high prevalence of PER-1 positive Acinetobacter spp. strains has been observed in Korea (54.6\%) [11]. Although microorganisms with PER-1 expression have been found to predominate in Turkey, outbreaks induced by $P$. aeruginosa strains have been reported from Italy [5], where PER-1 has also been detected in P. mirabilis and Alcaligenes faecalis [23, 24]. Likewise, the presence of this enzyme has also been noted in France and Spain [25, 26]. PER-1 


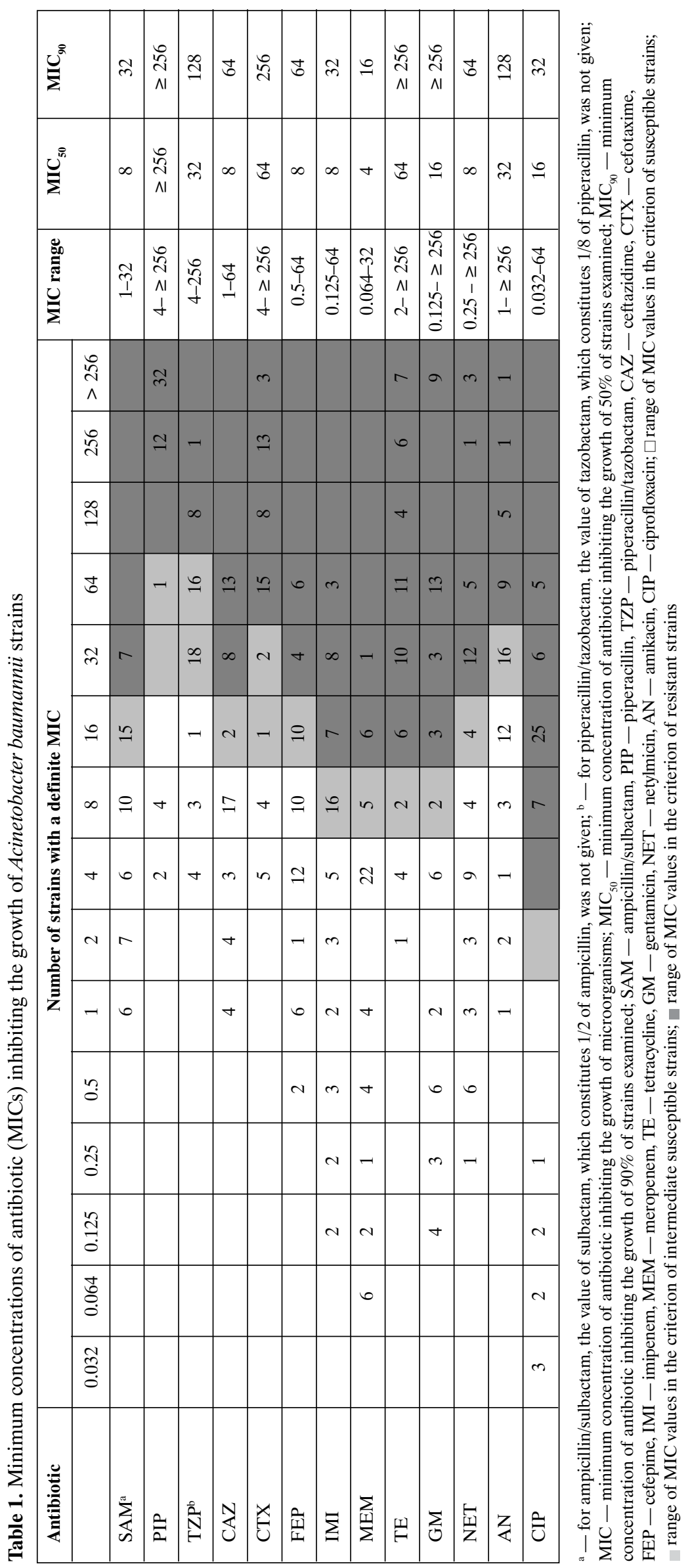


Table 2. Commonest phenotypes of resistance to antibiotics among the strains of Acinetobacter baumannii

\begin{tabular}{|l|l|l|l|l|l|l|l|l|l|l|l|l|l|l|}
\hline $\begin{array}{l}\text { Resistance to } \\
\text { a definite number } \\
\text { of antibiotics }\end{array}$ & \multicolumn{9}{|c|}{$\begin{array}{l}\text { Number } \\
\text { of strains }\end{array}$} \\
\hline 11 & $\mathrm{PIP}^{\mathrm{R}}$ & $\mathrm{TZP}^{\mathrm{R}}$ & $\mathrm{CAZ}^{\mathrm{R}}$ & $\mathrm{CTX^{ \textrm {R } }}$ & $\mathrm{FEP}^{\mathrm{R}}$ & $\mathrm{IMI}^{\mathrm{R}}$ & $\mathrm{MEM}^{\mathrm{R}}$ & $\mathrm{TE}^{\mathrm{R}}$ & $\mathrm{GM}^{\mathrm{R}}$ & $\mathrm{NET}^{\mathrm{R}}$ & & $\mathrm{CIP}^{\mathrm{R}}$ & 2 \\
\hline 8 & $\mathrm{PIP}$ & & & $\mathrm{CTX}^{\mathrm{R}}$ & & $\mathrm{IMI}^{\mathrm{R}}$ & & $\mathrm{TE}^{\mathrm{R}}$ & $\mathrm{GM}^{\mathrm{R}}$ & $\mathrm{NET}^{\mathrm{R}}$ & $\mathrm{AN}^{\mathrm{R}}$ & $\mathrm{CIP}^{\mathrm{R}}$ & 2 \\
\hline 5 & $\mathrm{PIP}^{\mathrm{R}}$ & & $\mathrm{CAZ}^{\mathrm{R}}$ & $\mathrm{CTX}^{\mathrm{R}}$ & & & & $\mathrm{TE}^{\mathrm{R}}$ & & & & $\mathrm{CIP}^{\mathrm{R}}$ & 6 \\
\hline 4 & $\mathrm{PIP}^{\mathrm{R}}$ & & & $\mathrm{CTX}^{\mathrm{R}}$ & & & & $\mathrm{TE}^{\mathrm{R}}$ & & & & $\mathrm{CIP}^{\mathrm{R}}$ & 3 \\
\hline 3 & $\mathrm{PIP}^{\mathrm{R}}$ & & & $\mathrm{CTX}$ & & & & & & & & $\mathrm{CIP}^{\mathrm{R}}$ & 2 \\
\hline 2 & & & & & & & & $\mathrm{TE}^{\mathrm{R}}$ & & & $\mathrm{AN}^{\mathrm{R}}$ & & 2 \\
\hline
\end{tabular}

PIP — piperacillin; TZP — piperacillin/tazobactam; CAZ — ceftazidime; CTX — cefotaxime; FEP — cefepime; IMI — imipenem; $\mathrm{MEM}$ - meropenem; TE — tetracycline; GM — gentamicin; NET — netylmicin; AN — amikacin; CIP — ciprofloxacin; ${ }^{\mathrm{R}}$ — resistant

producing Acinetobacter baumannii has been reported from France, Belgium, Romania, Russia and Hungary $[10,19,27-29]$. Subsequent variants have been described in the PER family in various bacteria (also in A. baumannii), among which PER-2 is found to predominate in some South American countries [30,31].

Most strains of $A$. baumannii with the $b_{\text {PER-1 }}$ gene, investigated in various countries, do not exhibit a consanguineous relationship to one another, something that is suggested by a clonal model of spread of this mechanism of resistance $[9,11]$. The analysis of the bla $a_{\text {PER-1 }}$ gene in various Gram-negative bacteria revealed not only (in most cases) their chromosomal, but also plasmid, location [32, 33]. These genes were identified within transposon Tn1213 limited by two insertion sequences ISPa12 and ISPa13, when they had chromosomal location in $P$. aeruginosa, $P$. stuartii and $A$. baumannii. Contrary to that, the PER-1 gene carried on plasmids was not part of the above-mentioned transposon - only the ISPa12 sequence above the $b l a_{\text {PER-1 }}$ gene was identified. These plasmids have been isolated from Salmonella enterica serowar Typhimurium and $A$. baumannii. The relation between $b l a_{\text {PER-2 }}$ genes also with the ISPa12 sequence seems to suggest a similar mechanism of gene mobilization on separate continents ( $b l a_{\mathrm{PER}-2}$ in South America vs. blaPER-1 in Europe and Asia). In both cases, the expression of the $b l a_{\text {PER-1 }}$ gene was controlled by the promoter sequence located in ISPa12. It is interesting that the $b l a_{\text {PER-3 }}$ gene detected in the Aeromonas punctata strain was combined with the ISCR1 element situated within class 1 integron In39. This finding may indicate a different mode of $b l a_{\mathrm{PER}}$ gene spread.

In Poland, ESBLs are seldom detected in other groups of bacteria (non-fermentative bacilli), apart from the Enterobacteriaceae family. Initial reports on PER-1 refer to $P$. aeruginosa strains [34], which, compared to our findings, indicates a low involvement of these enzymes in resistance to $\beta$-lactams in non-fermentative bacilli. The appearance of PER $\beta$-lactamases in new species of bacteria in various regions suggests the continuous spread of resistance determinants.

\section{Conclusions}

The application of phenotypic tests used to detect ESBL showed a likely presence of this mechanism of resistance in approximately $20 \%$ of Acinetobacter baumannii strains. Our strains seem not to use ESBL PER-1 as a determinant of resistance to $\beta$-lactam antibiotics. The most active antibiotics against the strains examined included meropenem, cefepim and ampicillin with sulbactam.

\section{References}

1. Gales AC, Jones RN, Forward KR, Linares J, Sader HS, Verhoef J. Emerging importance of multidrug-resistant Acinetobacter species and Stenotrophomonas maltophilia as pathogens in seriously ill patients: geographic patterns, epidemiological features, and trends in the SENTRY Antimicrobial Surveillance Program (1997-1999). Clin Infect Dis. 2001;32:104-113.

2. Perez F, Hujer AM, Hujer KM, Decker BK, Rather PN, Bonomo RA. Global challenge of multidrug-resistant Acinetobacter baumannii. Antimicrob Agents Chemoter. 2007;51:3471-3484.

3. Wieczorek P, Sacha P, Hauschild T, Źórawski M, Krawczyk M, Tryniszewska M. Multidrug resistant Acinetobacter baumannii - the role of AdeABC (RND family) efflux pump in resistance to antibiotics. Folia Histochem Cytobiol. 2008; 46:257-267.

4. Vahaboglu H, Ozturk R, Aygun G, Coskunkan F, Yaman A, Kaygusuz A, Leblebicioglu H, Balik I, Aydin K, Otkun M. Widespread detection of PER-1-type extended-spectrum $\beta$-lactamases among nosocomial Acinetobacter and Pseudomonas aeruginosa isolates in Turkey: a nationwide multicenter study. Antimicrob Agents Chemother. 1997;41:2265-2269.

5. Poirel L, Karim A, Mercat A, Le Thomas I, Vahaboglu H, Richard C, Nordmann P. Extended-spectrum $\beta$-lactamaseproducing strain of Acinetobacter baumannii isolated from a patient in France. J Antimicrob Chemother. 1999;43:157-158. 
6. Yong D, Shin JH, Kim S et al. High prevalence of PER-1 extended-spectrum $\beta$-lactamase-producing Acinetobacter spp. in Korea. Antimicrob Agents Chemother. 2003;47: 1749-1751.

7. Nordmann P, Ronco E, Naas T, Duport C, Michel-Briand Y, Labia R. Characterization of a novel extended-spectrum $\beta$-lactamase from Pseudomonas aeruginosa. Antimicrob Agents Chemother. 1993;37:962-969.

8. Nordmann P, Naas T. Sequence analysis of PER-1 extended-spectrum $\beta$-lactamase from Pseudomonas aeruginosa and comparison with class A $\beta$-lactamases. Antimicrob Agents Chemother. 1994;38:104-114.

9. Lahey Clinic. Available at http://lahey.org/Studies. Accessed November 13, 2011.

10. Claeys G, Verschraegen G, de Baere T, Vaneechoutte M. PER- $1 \beta$-lactamase-producing Pseudomonas aeruginosa in an intensive care unit. J Antimicrob Chemother. 2000; 45:924-925.

11. Pagani L, Mantengoli E, Migliavacca R et al. Multifocal detection of multidrug-resistant Pseudomonas aeruginosa producing the PER- 1 extended spectrum $\beta$-lactamase in Northern Italy. J Clin Microbiol. 2004;42:2523-2529.

12. Turton JF, Woodford N, Glover J, Yarde S, Kaufmann ME, Pitt TL. Identification of Acinetobacter baumannii by detection of the bla ${ }_{\text {OXA-5-like }}$ carbapenemase gene intrinsic to this species. J Clin Microbiol. 2006;44:2974-2976.

13. Jarlier V, Nicolas M, Fournier G, Philippon A. Extended broad-spectrum $\beta$-lactamases conferring transferable resistance to agents in Enterobacteriaceae: hospital prevalence and susceptibility patterns. Rev Infect Dis. 1988;10:867-878.

14. Pitout JDD, Reisbig MD, Venter EC, Church DL, Hanson ND. Modification of the double-disk test for detection of Enterobacteriaceae producing extended-spectrum and AmpC $\beta$-lactamases. J Clin Microbiol. 2003;41:3933-3935.

15. De Champs C, Poirel L, Bonnet R et al. Prospective survey of $\beta$-lactamases produced by ceftazidime-resistant Pseudomonas aeruginosa isolated in a French hospital in 2000. Antimicrob Agents Chemother. 2002;46:3031-3034.

16. European Committee for Antimicrobial Susceptibility Testing (EUCAST) of the European Society of Clinical Microbiology and Infectious Diseases (ESCMID). Determination of minimum inhibitory concentration (MICs) of antibacterial agents by broth dilution. Clin Microbiol Infect. 2003;9:1-7.

17. Clinical and Laboratory Standards Institute. Performance Standards for Antimicrobial Susceptibility Testing; Seventeenth Informational Supplement. CLSI document M100-S17. Wayne, Pennsylvania; 2007.

18. Yin XL, Hou TW, Xu SB et al. Detection of drug resistanceassociated genes of multidrug-resistant Acinetobacter baumannii. Microb Drug Resist. 2008;14:145-150.

19. Naas T, Bogaerts P, Bauraing C, Degheldre Y, Glupczynski Y, Nordmann P. Emergence of PER and VEB extended-spectrum $\beta$-lactamases in Acinetobacter baumannii in Belgium. J Antimicrob Chemother. 2006;58:178-182.

20. Endimiani A, Luzzaro F, Migliavacca R et al. Spread in an Italian hospital of a clonal Acinetobacter baumannii strain producing the TEM-92 extended-spectrum $\beta$-lactamase. Antimicrob Agents Chemother. 2007;51:2211-2214.

21. Sinha M, Srinivasa $H$, Macaden R. Antibiotic resistance profile and extended spectrum $\beta$-lactamase (ESBL) production in Acinetobacter species. Indian J Med Res. 2007;126:63-67.

22. Kolayli F, Gacar G, Karadenizli A, Sanic A, Vahaboglu H. PER-1 is still widespread in Turkish hospitals among Pseudomonas aeruginosa and Acinetobacter spp. FEMS Microbiol Lett. 2005;249:241-245.

23. Pereira M, Perilli M, Mantengoli E et al. PER-1 extended-spectrum $\beta$-lactamase production in an Alcaligenes faecalis clinical isolate resistant to expanded-spectrum cephalosporins and monobactams from a hospital in Northern Italy. Microb Drug Resist. 2000;6:85-90.

24. Pagani L, Migliavacca R, Pallecchi L, Matti C, Giacobone E, Amicosante G, Romero E, Rossolini GM. Emerging extended-spectrum $\beta$-lactamases in Proteus mirabilis. J Clin Microbiol. 2002;40:1549-1552.

25. Lartigue MF, Fortineau N, Nordmann P. Spread of novel expanded-spectrum $\beta$-lactamases in Enterobacteriaceae in a university hospital in the Paris area, France. Clin Microbiol Infect. 2005;11:588-591.

26. Miro E, Mirelis B, Navarro F et al. Surveillance of extended-spectrum $\beta$-lactamases from clinical samples and faecal carriers in Barcelona, Spain. J Antimicrob Chemother. 2005;56:1152-1155.

27. Naas T, Kernbaum S, Allali S, Nordmann P. Multidrug-resistant Acinetobacter baumannii, Russia. Emerg Infect Dis. 2007;13:669-671.

28. Naas T, Nordmann P, Heidt A. Intercountry transfer of PER-1 extended-spectrum $\beta$-lactamase-producing Acinetobacter baumannii from Romania. Int J Antimicrob Agents. 2007;29:223-232.

29. Szabo D, Szentandrassy J, Juhasz Z, Katona K, Nagy K, Rokusz L. Imported PER-1 producing Pseudomonas aeruginosa, PER-1 producing Acinetobacter baumannii and VIM2-producing Pseudomonas aeruginosa strains in Hungary. Ann Clin Microbiol Antimicrob. 2008;7:12.

30. Pasteran F, Rapoport M, Petroni A et al. Emergence of PER-2 and VEB-1a in Acinetobacter baumannii strains in the Americas. Antimicrob Agents Chemother. 2006;50:3222-3224.

31. Power P, Di Conza J, Rodriguez MM et al. Biochemical characterization of PER-2 and genetic environment of bla $\mathrm{PER}_{\text {-2 }}$. Antimicrob Agents Chemother. 2007;51:2359-2365.

32. Poirel L, Cabanne L, Vahaboglu H, Nordmann P. Genetic environment and expression of the extended-spectrum

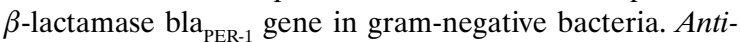
microb Agents Chemother. 2005;49:1708-1713.

33. Poirel L, Naas T, Nordmann P. Genetic support of extended-spectrum $\beta$-lactamases. Clin Microbiol Infect. 2008; 14:75-81.

34. Empel J, Filczak K, Mrówka A, Hryniewicz W, Livermore DM, Gniadkowski M. Outbreak of Pseudomonas aeruginosa infections with PER- 1 extended-spectrum $\beta$-lactamase in Warsaw, Poland: further evidence for an international clonal complex. J Clin Microbiol. 2007;45:2829-2834.

Submitted: 25 August, 2011

Accepted after reviews: 23 November, 2011 